\title{
Der Umfang der ärztlichen Dokumentationspflicht
}

\section{Roger König}

Dr. iur., LL.M., Rechtsanwalt

\author{
Gemäss der Ansicht des Bundesgerichts bezieht sich die Dokumentationspflicht \\ auf das medizinisch Notwendige und Übliche, ihr Zweck ist vorrangig die Behand- \\ lungssicherheit. Das Fehlen medizinisch nicht relevanter Aufzeichnungen kann \\ daher bei gerichtlichen Auseinandersetzungen keine beweisrechtlichen Nachteile \\ für den Arzt zur Folge haben.
}

Die Thematik der Arzthaftpflicht ist kontrovers und beschäftigt sowohl Mediziner als auch Juristen immer wieder. In seinem jüngsten Urteil befasste sich das Bundesgericht mit der ärztlichen Dokumentationspflicht. Dabei legte es den Umfang dieser Pflicht fest, der sich auf das medizinisch Notwendige und Übliche beschränkt. Nachfolgend wird zunächst der Fall [1] ${ }^{*}$ geschildert und sodann das Urteil erläutert. Anschliessend werden die wichtigsten Grundsätze der Arzthaftpflicht angemerkt und mit dem neuen Urteil in einen Gesamtkontext gesetzt.

\section{Der Fall}

Am 23. Juni 1993 brachte eine Frau ihr Kind zur Welt. Während des Geburtsvorgangs nahm der Gynäkologe einen medianen Dammschnitt («mediane Episiotomie») vor und beendete die Geburt mittels Saugglocke («Vakuumextraktion»). Bei der Geburt kam es zu einem Dammriss, der vom Gynäkologen unmittelbar nach der Geburt genäht wurde. In der Folge litt die Frau an einer Stuhlinkontinenz.

Die Frau machte gegenüber dem Gynäkologen geltend, dieser habe durch unsorgfältige Vorgehensweise bei ihr einen Riss des Schliessmuskels im Anus («Sphinkterriss») und dadurch eine heute nach wie vor bestehende Stuhlinkontinenz verursacht.

Im Jahr 2005 erhob die Frau Klage gegen den Gynäkologen und forderte von ihm eine Genugtuung in der Höhe von 100000 Franken. Die beiden kantonalen Instanzen (das Bezirksgericht Zürich und das Obergericht des Kantons Zürich) bejahten die Haftung des Gynäkologen und zwar im Umfang von 60000 Franken. Das Obergericht des Kantons Zürich begründete seinen Entscheid zu Lasten des Gynäkologen unter anderem damit, dass dieser nach der Geburt verpflichtet war, eine Rektaluntersuchung bei der Frau durchzuführen. Weil aber eine solche nicht dokumentiert sei, sei davon auszugehen, dass der Gynäkologe die Rektaluntersuchung nicht durchgeführt habe und deshalb die ärztliche Sorgfaltspflicht verletzt hätte.

\section{Das Urteil}

Auf Beschwerde des Gynäkologen hin hob das Bundesgericht mit Urteil vom 19. August 2015 den Entscheid des Obergerichts des Kantons Zürich auf [2]. Das Bundesgericht verneinte eine sogenannte Arzthaftpflicht und wies die Klage der Frau gegen den Gynäkologen vollumfänglich ab.

Besonders wichtig ist die Erwägung 5 des Urteils, die zur Publikation in der amtlichen Sammlung des Bundesgerichts vorgesehen und entsprechend als Leiturteil zu beachten ist [3]. Das Bundesgericht äusserte sich darin erstmals im Einzelnen zum Umfang der ärztlichen Dokumentationspflicht. 


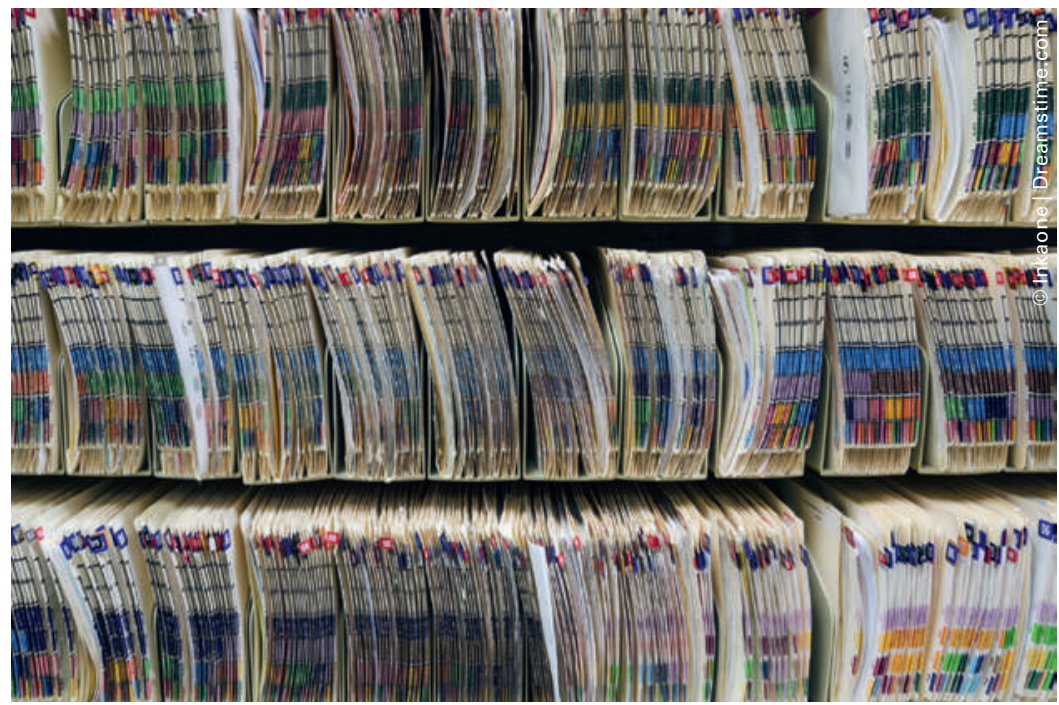

Das neue Leiturteil des Bundesgerichts schränkt die ärztliche Dokumentationspflicht auf einen vernünftigen Rahmen ein.

Unbestritten war im vorliegenden Fall, dass eine Rektaluntersuchung nach der Geburt nicht dokumentiert wurde.

In diesem Zusammenhang wies das Bundesgericht darauf hin: «Der Umfang der Dokumentationspflicht ergibt sich aus ihrem Zweck.» Entscheidend ist somit, was als Zweck der Dokumentationspflicht angesehen wird:

- In der juristischen Lehre finden sich (etwas überspitzt) fast so viele Zwecke wie Autoren. So soll die Dokumentation die korrekte Behandlung sicherstellen, namentlich auch bei der Behandlung durch mehrere Personen oder bei Arztwechsel. Mit ihr erfülle der Arzt seine auftragsrechtliche Rechenschaftspflicht und schliesslich diene sie auch der Beweissicherung [4].

- Gemäss der Ansicht des Bundesgerichts ist der Zweck der Dokumentation vorrangig die Behandlungssicherheit. So bestimmt sich das, was aufzuzeichnen ist, nach medizinischen Kriterien. Folglich kann das Unterbleiben medizinisch nicht relevanter Aufzeichnungen in juristischen Auseinandersetzungen keine beweisrechtlichen Nachteile für den Arzt zur Folge haben [5].

- Konkret ist nur, aber immerhin, das medizinisch Notwendige und Übliche zu dokumentieren. Diese Umschreibung sollte keinesfalls dazu verleiten, anzunehmen, die Anforderungen an die ärztliche Dokumentationspflicht seien tief. Vielmehr ist das Gegenteil der Fall: Im Sinne der Erwägung 5.1 des Bundesgerichts muss davon ausgegangen werden, dass in der Schweiz eine ebenso strenge Dokumentationspflicht gilt wie etwa in Deutschland. Jedoch ausgerichtet auf medizinische Üblichkeit und Erfor- derlichkeit und nicht zum Zweck, dem Patienten die Beweisführung zu sichern [6].

Von diesem Verständnis der Dokumentationspflicht ausgehend, sind (die im vorliegenden Fall interessierenden) Kontrolluntersuchungen dann nicht dokumentationspflichtig, wenn es medizinisch üblich ist, bei Ausbleiben eines positiven Befundes keine Aufzeichnungen vorzunehmen. In dieser Konstellation kann aus einer fehlenden Dokumentation nicht auf das Unterbleiben entsprechender Untersuchungen geschlossen werden [7]. Massgeblich war im eingangs geschilderten Fall somit die Frage, ob die Dokumentation der Rektaluntersuchung im Jahr 1993 aus medizinischen Gründen notwendig und üblich war. Diese Frage war selbstverständlich nicht von einem Juristen, sondern von einem Arzt im Rahmen eines medizinischen Gutachtens zu beantworten. Der damit beauftragte Arzt stellte in seinem Gutachten fest, dass die Rektaluntersuchung zur Standarduntersuchung gehöre und dass diese Untersuchung im Partogramm nicht dokumentiert sei. Jedoch sei es nicht absolut üblich und gefordert, dass die Rektaluntersuchung dokumentiert werde. Diese medizinische Beurteilung des Gutachters führte im Ergebnis dazu, dass die Frau dem Gynäkologen keine ärztliche Sorgfaltspflichtverletzung wegen nicht durchgeführter Rektaluntersuchung nachweisen konnte [8]. Wäre der Gutachter zum gegenteiligen Schluss gekommen, und hätte er die Notwendigkeit und Üblichkeit der Dokumentation der Rektaluntersuchung bejaht, hätte das Bundesgericht die Haftung des Gynäkologen wohl bestätigt. Das zeigt, wie wichtig es ist, dass die Ärzteschaft den Umfang der Dokumentationspflicht (der sich auf das medizinisch Notwendige und Übliche erstreckt) kennt und sicherstellt, dass diese Pflicht eingehalten wird.

\section{Anmerkungen}

Die Rechtsbeziehung zwischen Arzt und Patient gilt als Auftragsvertrag [9]. Im Rahmen dieses Vertrages schuldet der Arzt dem Patienten ein getreues und sorgfältiges Tätigwerden. Das bedeutete im konkreten Fall, der Gynäkologe schuldete nicht etwa einen komplikationslosen und verletzungsfreien Geburtsvorgang, sondern eine sorgfältige ärztliche Behandlung der Frau während dieses Vorgangs.

Die Haftung des Arztes (wie im Übrigen auch die des Anwalts) erstreckt sich auf die getreue und sorgfältige Ausführung des ihm übertragenen Auftrags [10]. Die vom Arzt geschuldete Sorgfalt lässt sich nicht abschliessend festlegen, sondern sie richtet sich nach den Umständen des Einzelfalls [11]. 
- Ein wichtiger Bestandteil der ärztlichen Vertragspflicht ist die Aufklärungspflicht [12]. Deren Befolgung soll sicherstellen, dass der Patient in Kenntnis der Sachlage seine Einwilligung in eine ärztliche Behandlung gibt [13]. Die Beweislast für eine hinreichende Aufklärung liegt beim Arzt [14]. Entsprechend sollte der Arzt unbedingt schriftlich dokumentieren, dass er den Patienten hinreichend aufgeklärt hat.

- Weiter trifft den Arzt eine vertragliche Sorgfaltspflicht bei der Stellung der Diagnose. Dabei hat der Arzt die Pflicht, die Untersuchung sorgfältig, nach den Regeln der Kunst und dem jeweiligen Stand der Wissenschaft vorzunehmen [15]. Zu beachten ist, dass in der Unrichtigkeit der Diagnose für sich allein noch kein Verschulden des Arztes liegt. Ist der Arzt bei der Vornahme der Diagnose gewissenhaft vorgegangen, hat er also die erforderliche Zeit und Aufmerksamkeit für die Untersuchung und Befragung des Patienten aufgewendet und die gebotenen medizinischen Abklärungen getroffen, so kann ihm keine Nachlässigkeit oder Unvorsichtigkeit vorgeworfen werden und eine Haftung fällt ausser Betracht [16].

- Selbstverständlich auch von der ärztlichen Sorgfaltspflicht umfasst ist die Durchführung der Behandlung. Ein Behandlungsfehler (gemeinhin auch Kunstfehler [17] genannt) besteht darin, dass der Arzt eine falsche Behandlung durchführt oder zwar die richtige Behandlung wählt, diese aber falsch ausführt. Jedoch kann aus einer Behandlung, die sich nachträglich als unangemessen oder sogar als verfehlt erweist, nicht ohne Weiteres auf eine Haftung geschlossen werden. Sie ist vielmehr nur dann zu bejahen, wenn eine Behandlung nach dem anerkannten und bekannten Wissensstand der Medizin nicht mehr als vertretbar erscheint, mithin ausserhalb der objektivierten ärztlichen Kunst steht [18].

Macht ein Patient gegenüber dem Arzt eine vertragliche Sorgfaltspflichtverletzung geltend, die auf einen angeblichen Behandlungsfehler zurückzuführen ist, so stellt sich die Frage, wer diese Vertragsverletzung beweisen muss. In diesem Zusammenhang kann nun, wie das eingangs erwähnte Leiturteil aufzeigt, dem Umfang der ärztlichen Dokumentationspflicht eine entscheidende Bedeutung zukommen:

- Nach den allgemeinen Grundsätzen ist die Sorgfaltspflichtverletzung vom Geschädigten, also vom Patienten, zu beweisen [19]. Das heisst, im vorliegenden Fall hätte die Frau beweisen müssen, dass der Gynäkologe keine bzw. eine unsorgfältige Rektaluntersuchung durchgeführt hatte [20].
- In gewissen Fällen werden der beweisbelasteten Person Beweiserleichterungen zugestanden oder eine Sorgfaltspflichtverletzung wird sogar vermutet (Letzteres führt zu einer Umkehr der Beweislast zu Lasten des Arztes; eine solche Umkehr wurde vom Bundesgericht in einem älteren Leiturteil auch schon bejaht, später aber stark eingeschränkt [21]). Vorliegend verneinte das Bundesgericht sowohl eine Umkehr der Beweislast als auch eine Herabsetzung des Beweismasses zu Lasten des Arztes. In Bezug auf die hier interessierende Dokumentationspflicht wies das Bundesgericht aber darauf hin, dass eine Verletzung der (medizinisch notwendigen) Dokumentation eine Beweiserleichterung zu Lasten des Arztes und zu Gunsten des Patienten zur Folge haben kann [22]. Eine solche Beweiserleichterung kann dazu führen, dass das Gericht zum Schluss gelangt, eine Untersuchung sei nicht durchgeführt worden, da diese in der Dokumentation fehlt.

Das zeigt, wie wichtig es ist, dass die Ärzteschaft den Umfang der Dokumentationspflicht kennt.

Das neue Leiturteil des Bundesgerichts schränkt die ärztliche Dokumentationspflicht auf einen vernünftigen Rahmen ein, nämlich auf das, was medizinisch üblich und erforderlich ist. Mit anderen Worten: $\mathrm{Zu}$ dokumentieren ist das medizinisch Notwendige und Übliche. Was das genau ist, muss im Streitfall ein medizinischer Experte festlegen.

Selbst wenn der Experte im Einzelfall zum Schluss gelangen würde, die Dokumentation einer Untersuchung oder einer Behandlung wäre medizinisch notwendig und üblich gewesen, heisst das noch nicht, dass der Arzt auch effektiv haftet. Diesem bleibt selbstverständlich die Möglichkeit, Beweise vorzubringen, die unter Umständen auf eine Untersuchung oder eine Behandlung schliessen lassen (zum Beispiel mittels Zeugen oder einer vom Patienten vorbehaltlos bezahlten Rechnung). Um nicht auf diese unsicheren «Notnägel» zurückgreifen zu müssen, empfiehlt es sich, den zuvor skizzierten Umfang der Dokumentationspflicht zu kennen und dieser Pflicht, das medizinisch Notwendige und Übliche zu dokumentieren, bewusst nachzukommen.

\section{Danksagung}

Herrn Dr. iur. Bruno Pasquier, Rechtsanwalt, danke ich herzlich für die Übersetzung der Zusammenfassung in die französische Sprache. 


\section{Referenzen}

1 Vgl. Urteil des Bundesgerichts 4A_137/2015 vom 19. August 2015, Sachverhalt lit. A-D.

2 Vgl. Urteil des Bundesgerichts 4A_137/2015 vom 19. August 2015, E. 1-10 (E. 5 ist als Leitentscheid zur Publikation in der amtlichen Sammlung des Bundesgerichts vorgesehen).

3 Die Publikation in der amtlichen Sammlung des Bundesgerichts dauert mehrere Wochen und ist noch nicht erfolgt. Das ganze Urteil, das auch die wichtige Erwägung 5 umfasst, ist online abrufbar unter: www.bger.ch $\rightarrow$ Rechtsprechung $\rightarrow$ Rechtsprechung gratis; Weitere Urteile ab 2000; 4A_137/2015 im Textfeld eingeben und Klick auf Suchen)

4 Vgl. Urteil des Bundesgerichts 4A_137/2015 vom 19. August 2015, E. 5.1 (m.w.H.)

5 Vgl. Urteil des Bundesgerichts 4A_137/2015 vom 19. August 2015, E. 5.1 mit Hinweis auf Susanne Bollinger Hammerle, Die vertragliche Haftung des Arztes für Schäden bei der Geburt, 2004, S. 156

6 Vgl. Urteil des Bundesgerichts 4A 137/2015 vom 19. August 2015, E. 5.1 (m.w.H.).

7 Urteil des Bundesgerichts 4A 137/2015 vom 19. August 2015, E. 5.1 (letzter Absatz).

8 Vgl. Urteil des Bundesgerichts 4A_137/2015 vom 19. August 2015, E. $5.3 \mathrm{ft}$

9 Vgl. Walter Fellmann, Der einfache Auftrag Art. 394-406 OR, Berner Kommentar VI/2/4, 1992, OR 394 N 185.

10 Vgl. Urteil des Bundesgerichts 4A_137/2015 vom 19. August 2015, E. 4.

11 Vgl. Walter Fellmann, a.a.O. [Fn. 9], OR 398 N 384.

12 Vgl. BGE 133 III 121, E. 4.1.2.
13 Vgl. BGE 117 Ib 197, E. 3b

$14 \mathrm{Vgl}$. Antoine Roggo/Daniel Staffelbach, Offenbarung von Behandlungsfehlern, in: AJP/PJA 4/2006, S. 415

15 Vgl. BGE 64 II 200, E. 4 a

16 Vgl. BGE 57 II 196, E. 3; vgl. auch Frédéric Krauskopf, Skript Vertragsrecht (Modul Privatrecht), 2011, S. 59.

17 Der Begriff des Kunstfehlers ist ungenau und deckt sich nicht vollumfänglich mit dem des Behandlungsfehlers. Wie Roggo/ Staffelbach in einem wissenschaftlichen Beitrag zutreffend aufgezeigt haben, fällt der Kunstfehler zwar unter den Begriff des Behandlungsfehlers. Jedoch gibt es auch Behandlungsfehler, die keine Kunstfehler sind. So zum Beispiel die unzureichende Aufklärung zur Therapiesicherung oder die mangelhafte Eingriffsaufklärung (zum Ganzen vgl. Antoine Roggo/Daniel Staffelbach, a.a.O. [Fn. 14], S. 414)

18 Frédéric Krauskopf, a.a.O. [Fn. 16], S. 59; vgl. Urteil des Bundesgerichts 6S.127/2003 vom 28. November 2003, E. 3.3 f.; vgl. auch BGE 130 IV 7, E. 3.3.

19 BGE 133 III 121, E. 3.4; Urteil des Bundesgerichts 4A 137/2015 vom 19. August 2015, E. 5.2 und 6.3.1. (Satz 1); vgl. auch Urteil des Bundesgerichts 4C.53/2000 vom 13. Juni 2000, E. 2a (erster Satz m.w.H.).

20 Urteil des Bundesgerichts 4A 137/2015 vom 19. August 2015, E. 6.3.1 (Satz 2)

21 Urteil des Bundesgerichts 4A 137/2015 vom 19. August 2015, E. 6.3.1 (Abs. 2 f. m.w.H. auf BGE 120 II 248, E. 2c; Urteil des Bundesgerichts 4C.53/2000 vom 13. Juni 2000, E. 2b; und Jörg Schmid, Die privatrechtliche Rechtsprechung des Bundesgerichts im Jahr 2006 und 2007, ZBJV 146/2010, S. 615 ff.).

22 Urteil des Bundesgerichts 4A_137/2015 vom 19. August 2015, E. $5.2 \mathrm{ff}$ 